\title{
ETUDE DE BASE ENTOMOLOGIQUE EN PRELUDE A UNE CAMPAGNE DE LUTTE CONTRE LA TRYPANOSOMIASE DANS LES CERCLES DE KADIOLO ET SIKASSO AU MALI.
}

\section{ENTOMOLOGICAL BASIS STUDY IN PRELUDE TO THE FIGHTING CAMPAIGN AGAINST TRYPQNOSOMIASIS IN THE DISTRICTS OF KADIOLO AND SIKASSO (MALI)}

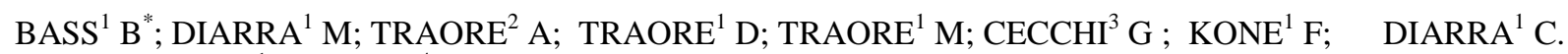

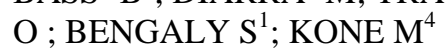 \\ ${ }^{1}$ : Laboratoire Central Vétérinaire, Bamako, Mali \\ 2: Université des Sciences, des Techniques et des Technologies de Bamako, Mali \\ ${ }^{3}$ : FAO Sub regional Office for Eastern Africa, Addis Ababa, Ethiopia \\ 4: TCP/MLI/3402 FAO \\ * Auteur correspondant : Boubacar BASS, entomologie, Laboratoire Central Vétérinaire. BP. 2295, Bamako, \\ Mali Tél. : + 22320243344 ; Email : drbassml1@ yahoo.fr
}

\begin{abstract}
Résumé
Le projet TCP/MLI/3402 FAO intitulé « Appui à la lutte contre la mouche tsé-tsé et les trypanosomoses dans les cercles de Sikasso et Kadiolo, au Mali » a été crée pour appuyer le Projet de Lutte contre la Mouche tsé-tsé et les trypanosomoses (PLMT) dans ses efforts de contrôle des tsé-tsé et trypanosomes. Son objectif était de créer les conditions amenant l'état, les collectivités territoriales et les communautés villageoises à prendre en charge la lutte contre la trypanosomose et ses vecteurs de façon durable. Dans les cercles de Kadiolo (20 villages) et Sikasso (15 villages) 35 villages ont été prospectés. Pour cette étude 350 pièges biconiques Challier Laveissière ont été posés dans les galeries et au niveau des points de contact des différents cours d'eau et géo référencés à l'aide de GPS. La prospection entomologique dans la zone de l'étude a permis la capture de deux espèces de glossines: Glossina palpalis gambiensis et Glossina tachinö̈des. Le nombre total de glossines capturées est 786 dont 405 mâles et 381 femelles. Parmi les glossines capturées, il ya 691 Glossina palpalis gambiensis dont 349 mâles et 342 femelles, 95 Glossina tachinoïdes dont 56 mâles et 39 femelles. Le sexe ratio est de 1,12, donc légèrement en faveur des mâles. Le taux d'infection des glossines qui concerne le cercle de Kadiolo seulement est $0 \%$. La Densité Apparente de glossines par Piège et par jour (DAP) dans la zone du projet est 2,2.
\end{abstract}

Mots clés : Prospections, glossines, lutte, Mali.

\begin{abstract}
The project TCP/MLI/3402 FAO called $<<$ fighting support against the tsé-tsé flies and trypanosomiasis in the prefecture of Sikasso and Kadiolo in Mali > was created for support the project of fighting against flies tse-tse and trypanosomiasis (PLMT) in their effort to control the flies' tse-tse and trypanosomiasis. His object was to create the conditions to allow government, territorials' community and village's community to take charge of the fighting against trypanosomiasis in the lasting way. In prefecture of Kadiolo (20 villages) and Sikasso (15 villages) 35 villages were prospected. For this study 350 traps biconics Challier-Laveissiere have been put down in the gallery and near the contacts points of different rivers which were located by GPS. Entomological prospecting in the study area was allowed to catch two species fly: Glossina palpalis gambiensis and Glossina .tachinoïdes. 786 flies were captured, among which 40 males and 381 females, 95 Glossina tachinoïdes among which 56 males and 39 females. The ratio-sex is 1.12, so slightly in favor of male. The infection rate of the flies in the prefecture of Kadiolo is $0 \%$. The apparent density of flies by trap per day (DAP) in the area of project is 2.2
\end{abstract}

Keywords: prospecting, glossinas, fight, Mali.

\section{Introduction}

Les glossines sont des Diptères Brachycères Cyclorrhaphes de la famille des Glossinidae avec un seul genre : Glossina. Elles diffèrent des autres mouches par l'adaptation de leurs pièces buccales à la piqûre ; à la différence de quelques autres insectes hématophages (moustiques, taons), les deux sexes se nourrissent de sang. Les glossines sont des mouches allongées, robustes, de coloration brun noirâtre à brun testacé, jamais métallique ; leur 
longueur sans la trompe, est comprise entre 6 et 16 $\mathrm{mm}$. Leur poids varie de 7 à $14 \mathrm{mg}$ chez Glossina tachinoïdes à 20 - $25 \mathrm{mg}$ chez Glossina morsitans (1). Ces insectes transmettent à l'homme et aux animaux domestiques, plusieurs espèces d'un protozoaire flagellé du genre Trypanosoma qui vit dans les liquides biologiques et les tissus des hôtes provoquant une maladie aiguë. Par contre certains mammifères sauvages (antilopes, girafes, buffles, lions, éléphants...) et quelques races d'animaux domestiques (taurins, N'dama, Baoulé, Lagune...) vivent, produisent et se reproduisent dans les zones infestées, bénéficiant d'une trypanotolérance. Ce serait peut être également le cas de certaines populations humaines des zones très humides.

La distribution de l'insecte est liée à son habitat : la végétation au bord des cours d'eaux, des lacs, des forêts - galeries et des vastes étendues de savane arbustive. (2) Au Mali les glossines occupent une superficie de $240000 \mathrm{~km}^{2}$ à très grand potentiel agricole. La région de Sikasso est infestée à $100 \%$, la région de Kayes à 76\%, la région de Koulikoro à $60 \%$ et la région de Ségou à $44 \%$ (3). Il est alors impératif de lutter contre cette maladie. L'un des moyens de contrôle de cette maladie est la lutte contre les vecteurs (4). La glossine, agent majeur de transmission des trypanosomoses animales et humaines est une contrainte pour le développement de l'agriculture et de l'élevage au Mali. Les activités de suppression des populations de glossines par l'installation de pièges et d'écrans imprégnés et le traitement épi cutané des animaux domestiques par des insecticides synthétiques importés et très onéreux, sont à la charge des communautés rurales. La présence des glossines en Afrique explique la faible production agricole à cause de l'affaiblissement des animaux malades, et l'invalidité des personnes touchées. De 1962 à la mise en place de ce projet en 2012, différents programmes et projets de recherche et de lutte contre les mouches tsé-tsé et les trypanosomoses ont été réalisés au Mali. Par exemple :

- Essais expérimentaux pour étudier l'efficacité de plusieurs molécules d'insecticides rémanents dans les galeries forestières en bordure des cours d'eau contre Glossina tachinoides (1972 - 1973).

- Lutte intégrée contre la mouche tsé-tsé au Mali à l'aide de la Technique de l'Insecte mâle Stérile (1991-1992).

Plus de $70 \%$ de la population malienne a comme activité principale l'agriculture. Au mali, l'élevage occupe la troisième place des produits d'exportation après le coton et l'or. Lutter contre les glossines, c'est augmenter les surfaces cultivables et les productions agricoles, donc réduire la pauvreté rurale et améliorer la sécurité alimentaire au Mali.
Préalablement aux activités de lutte dans la zone du projet TCP/MLI/3402 FAO, une étude qui avait pour but de définir la situation de référence à travers la collecte, le traitement et l'analyse des données de base entomologique a été confiée au Laboratoire Central Vétérinaire.

\section{Matériels et méthodes}

Sites d'étude :

Le cercle de Kadiolo couvre une superficie de 5375 $\mathrm{Km} 2$. Il est situé à l'extrême sud du Mali, limité à l'Est par le Burkina-Faso, à l'Ouest et au Sud par la RCI), au Nord par le cercle de Sikasso et au Nord-Ouest par le cercle de Kolondiéba. (5) Le cercle de Sikasso couvre une superficie de 19.100 $\mathrm{km}^{2}$. Il est limité au Nord par les Cercles de Koutiala et Dioïla, au Sud par le Cercle de Kadiolo, à l'Ouest par les Cercles de Kolondiéba et Bougouni, à l'Est par le Burkina-Faso. (5) Dans le cercle de Kadiolo, l'étude a concerné les villages mentionnés dans le tableau 1 Dans le cercle de Sikasso, l'étude a concerné les villages mentionnés dans le tableau 2.

Matériels :

Loupe binoculaire, piège biconique Challier Laveissière, Garmin $®$, microscope, boite de Pétri, lames et lamelles, trousse de dissection, solution physiologique

Méthodes:

La méthode de prospection préconisée pour cette étude fait appel à 350 pièges biconiques

Challier - Laveissière (6) très attractif pour les trois espèces rencontrées au Mali (Glossina palpalis gambiensis ; Glossina morsitans submorsitans ; Glossina tachinoides). Les pièges biconiques sont géo référencés et placés tous les jours avant 8 heures à côté de l'eau, de la végétation et au soleil pour les espèces ripicoles et en savane pour l'espèce savanicole. La distance entre deux pièges consécutifs à quelques exceptions (absence de végétation, absence de rayons solaires) est de $200 \mathrm{~m}$ environ, celle entre le piège et le sol est environ 10 $\mathrm{cm}$.

Le lendemain matin les pièges sont enlevés. Dans chaque cage (une partie du piège biconique Challier - Laveissière) une étiquette a été placée qui porte : la date, la localité, le numéro (point de capture). Après le dénombrement des différentes espèces capturées, les glossines sont séparées par sexe et les résultats sont portés sur l'étiquette. Au cours de nos captures, d'autres mouches piqueuses qui transmettent la trypanosomose telles les tabanidés et les stomoxes sont identifiées. Les résultats sont aussi portés sur l'étiquette. La dissection des glossines concerne l'appareil piqueur, le tube digestif et les glandes salivaires, organes de 
développement des parasites pathogènes pour les bovins (7). Après avoir été individualisés, ces organes sont examinés au microscope pour rechercher la présence de trypanosomes afin de déterminer le taux d'infection des glossines.

Le sexe ratio (rapport entre mâles et femelles) des glossines est déterminé dans chaque localité. Pour les enquêtes entomologiques, à chaque sortie l'équipe constituée comprend :

- Un Chercheur spécialiste des tsé-tsé, chargé de la supervision de l'équipe sur le terrain.

- Un technicien spécialisé en entomologie chargé de la pose et du relevé des pièges, de noter l'espèce et le sexe des glossines capturées, de noter l'espèce des vecteurs mécaniques de la trypanosomose capturés, de disséquer quelques glossines capturées.

\section{Résultats}

Distribution et DAP des glossines

Dans le cercle de Kadiolo, 200 pièges biconiques Challier - Laveissière ont été posés et géo référencés, deux espèces de glossines capturées : Glossina palpalis gambiensis et Glossina tachinoïdes. Au total, 402 glossines sont capturées dont 335 Glossina palpalis gambiensis et 67 Glossina tachinoïdes. Le nombre de Glossina palpalis gambiensis capturé dans les villages concernés par l'étude dans le cercle de Kadiolo est indiqué par la figure 1. Parmi les Glossina palpalis gambiensis capturés il ya 167 mâles et 168 femelles. Les captures les plus importantes pour Glossina palpalis gambiensis ont été observées à Gninasso (41 glossines), Zekoun et Galamakourou (39 glossines), Nimbougou (34 glossines). Par contre, à Katele et Dioumantene 0 glossine capturée. Le plus grand nombre de mâles de Glossina palpalis gambiensis a été capturé à Gninasso (29 glossines) et le plus grand nombre de femelles à Zekoun (25 glossines). Le nombre de Glossina tachinoides capturé dans les différents villages de l'étude dans le cercle de Kadiolo est indiqué par la figure 2. Parmi les Glossina tachinoïdes capturés dans le cercle de Kadiolo il ya 32 mâles et 35 femelles. Le plus grand nombre a été capturé à Zekoun (13 glossines), la capture est nulle dans beaucoup de villages (Katele, Dioumantene, Niérouani, Sieou-Kourani, Nimbougou, Siranikoroba, Daoulasso). Dans le cercle de Sikasso, 150 pièges biconiques Challier Laveissière ont été posés et leurs emplacements géoréferencés dans 15 villages. Deux espèces de glossines sont capturées: Glossina palpalis gambiensis et Glossina tachinoïdes. Au total, 384 glossines sont capturées dont 356 Glossina palpalis gambiensis et 28 Glossina tachinö̈des. Dans le cercle de Sikasso, 182 mâles et 174 femelles de Glossina palpalis gambiensis sont capturés. Des captures importantes sont observées à Diassadiè (59 glossines), Farako (56 glossines), Finibougou (51 glossines). Parmi les glossines capturées il ya 24 mâles et 4 femelles de Glossina tachinoüdes. Le nombre le plus élevé a été enregistré à Farako (6 glossines) et des captures nulles à Nagnasso, Pitagalasso, Konzasso Diassa, Tiogola, Ouahibera, Kafela. Au total dans la zone du projet TCP/MLI/3402, 350 pièges biconiques Challier Laveissière ont été posés dans les galeries et au niveau des points de contact des différents cours d'eau et géo référencés à l'aide de GPS. La prospection entomologique dans la zone de l'étude a permis la capture de deux espèces de glossines : Glossina palpalis gambiensis et Glossina tachinö̈des. Au total, 786 glossines sont capturées dont 405 mâles et 381 femelles. Parmi les glossines capturées, il ya 691 Glossina palpalis gambiensis dont 349 mâles et 342 femelles, 95 Glossina tachinö̈des dont 56 mâles et 39 femelles. Dans le cercle de Kadiolo, les plus fortes densités sont observées à Zekoun $(5,2)$ et les plus faibles à Katele (0) et Dioumantene (0). Dans le cercle de Sikasso, les plus fortes densités sont observées à Diassadiè $(6,6)$ et les plus faibles à Pitagalasso $(0)$. Les DAP les plus élevées ont été obtenues à Zekoun $(5,2)$, Mandela (5,3), Finibougou (5,4), Farako $(6,2)$, Diassadiè $(6,6)$ tandis que les plus faibles sont enregistrées à Katele (0), Dioumatene (0), Pitagalasso (0), Goloniena $(0,2)$, Fanidiama $(0,4)$, Nagnasso $(0,5)$, Nafegué $(0,7)$, M'Belasso $(0,8)$ et Zégoua $(0,9)$. La DAP dans la zone d'étude est 2,2. Taux d'infection des glossines

Pour des problèmes logistiques, la dissection a concerné le cercle de Kadiolo seulement. Le nombre des glossines disséquées est 175 et le taux d'infection $0 \%$. La dissection a concerné le tube digestif et le proboscis des glossines.

Sexe ratio des glossines capturées

A l'éclosion, le rapport mâles/femelles est voisin de 1 chez la plupart des espèces élevées au laboratoire, sauf pour Glossina morsitans submorsitans où il y a une distorsion génétique en faveur des femelles (68\% de femelles dans les insectariums du CIRDES). Celle-ci serait contrôlée par un facteur lié au chromosome X. (4)

Dans la nature, le sexe ratio est de 1 à l'éclosion, mais du fait de la plus grande longévité des femelles, il est en général en faveur des femelles lors de capture. Mais il existe des variations en fonction du lieu, de l'heure du jour, de la saison, de l'espèce et du moyen de capture. Dans cette étude le sexe ratio est de 1,12, donc légèrement en faveur des mâles. Le sexe ratio diffère entre deux points de 
capture mais en suivant les mêmes variations. Une analyse des résultats a permis de mettre en évidence que la proportion des sexes varie en fonction d'un complexe de facteurs qui comprend le sexe de la mouche (comportement), le captureur (adresse, comportement), la végétation (visibilité variable au cours des saisons) et la saison. Les femelles sont moins actives que les mâles alors qu'elles sont en plus grand nombre en raison de leur plus grande longévité que celle des mâles.

Vecteurs mécaniques capturés

Les tableaux 4 et 5 montrent la présence des vecteurs mécaniques (tabanidés et stomoxes) dans la zone du projet. Le plus grand nombre de vecteurs mécaniques a été capturé à Bananso, 17 Tabanus gratus. Dans beaucoup de localités (25 localités / 35), la présence des vecteurs mécaniques n'a pas été signalée. Ces vecteurs mécaniques permettent un relais de la transmission de Trypanosoma vivax.

\section{Discussion}

Contrairement à notre étude où deux espèces de glossines (Glossina palpalis gambiensis et Glossina tachinoides) ont été capturé, trois espèces de glossines (Glossina palpalis gambiensis, Glossina tachinoides et Glossina morsitans submorsitans) capturées en 1997 (3). Ceci peut s'expliquer par l'impact du changement global et surtout la rareté de la faune sauvage dont la présence de l'espèce Glossina morsitans submorsitans dépend largement. Une seule espèce de glossine a été capturée dans le bassin du Bani : Glossina palpalis gambiensis (8). L'étude menée dans le cercle de Bougouni en 2014 (9) confirme nos résultats où sur la base des résultats des prospections entomologiques, 3421 glossines sont capturées : parmi les glossines capturées, 80\% (2740/3421) sont de l'espèce Glossina palpalis gambiensis et $20 \%(681 / 3421)$ de l'espèce Glossina tachinoüdes. Cette ressemblance des résultats peut s'expliquer par l'appartenance des deux milieux d'études à la même zone éco - climatique. La DAP dans la zone d'étude est 2,2. Ces densités sont inférieures à celles obtenues par d'autres auteurs (8) en 2010 dans le bassin du Bani (5,6,7). Dans la zone de Sidéradougou au Burkina Faso en 2005 (12), une densité apparente de 13,7 a été obtenue. Le projet PATTEC Mali en janvier 2014 (non publié) a obtenu dans le bassin du Banifing (cercle de Koutiala) une DAP de 29. Mais le projet a évolué dans trois villages seulement (Tiby, Nampropele, Kéleni). Le taux d'infection des glossines s'élève à $10 \%$ des glossines disséquées dans le bassin du Bani (8). En Côte d'Ivoire, le taux d'infection obtenu s'élève à $28 \%$ (10) .Ces résultats ne corroborent pas ceux de notre étude. En Côte
d'Ivoire, les femelles sont plus infectées que les mâles, probablement parce qu'elles prennent un plus grand nombre de repas à intervalles rapprochés que les mâles, et parce que leur durée de vie est supérieure. Elles ont plus d'occasions de s'infecter et donc de transmettre un plus grand nombre de fois le parasite. Nos résultats confirment ceux de l'étude menée dans le bassin du Bani (8) où le sexe ratio est 1,05 . Nos résultats sont aussi semblables avec ceux obtenus dans le bassin versant de l'Oti au nord du Togo (11). Ceci peut s'expliquer par l'utilisation de la même technique de piégeage. Le sexe ratio est très variable au cours de l'année. Dans la forêt classée du KOU, le pourcentage des femelles capturées peut descendre au-dessous de 20 p. 100, mais peut aussi atteindre 50 p. 100. Le maximum se trouve durant les mois pluvieux ; un second maximum a lieu vers février et parfois mars (4). Le nombre de vecteurs mécaniques capturé est plus important dans l'étude menée dans le bassin du Bani (8). Cet écart important peut s'expliquer par les meilleures conditions écologiques pour les vecteurs mécaniques dans le bassin du Bani.

\section{Conclusion}

L'objectif de notre étude était de définir une situation de référence des mouches tsé-tsé avant les activités de lutte. Des prospections entomologiques ont permis d'identifier deux espèces de glossines dans la zone du projet: Glossina palpalis gambiensis et Glossina tachinö̈des. L'espèce Glossina morsitans submorsitans habituellement existante dans la zone du projet n'a pas été capturée. En plus des glossines, d'autres insectes vecteurs de la Trypanosomose Animale Africaine aussi ont été identifiés : tabanidés et stomoxes. Les DAP plus élevées ont été obtenues à Diassadiè $(6,6)$ et Farako $(6,2)$ et les nulles à Katele (0) et Pitagalasso (0). Le sexe ratio est légèrement en faveur des mâles. La dissection des glossines, qui a concerné le cercle de Kadiolo seulement a donné un taux d'infection de $0 \%$. En comparaison avec les résultats de 1997 (3), Il ressort clairement que la densité des glossines diminue davantage dans la zone du projet. Ce qui peut s'expliquer par les effets de sécheresse, du changement climatique et aussi l'anthropisation. En perspective, il serait judicieux qu'une étude soit menée au Mali afin de confirmer l'hypothèse que les vecteurs mécaniques de la trypanosomose permettent un relais de la transmission de Trypanosoma vivax.

\section{Remerciements}

L'équipe de recherche remercie la direction du Laboratoire Central Vétérinaire. 
Le Laboratoire Central Vétérinaire adresse ses sincères remerciements à la coordination du TCP/MLI/3402 pour sa franche collaboration. Les remerciements s'adressent également à la représentation de la FAO au Mali.

Le Laboratoire Central Vétérinaire remercie aussi le Projet FAO «Improving food security in sub Saharan Africa by supporting the progressive reduction of tsetse transmitted trypanosomosis in the framework of the NEPAD. (GTFS/RAF/474/ITA), financé par le gouvernement italien. Remerciements particuliers aux secteurs vétérinaires de Kadiolo et Sikasso, aux mandataires sanitaires des localités concernées. Sincères remerciements aux notabilités, aux guides.

\section{Références}

1. ITARD J, 1986. Les glossines ou mouches tsétsé. ENV, Maisons Alfort, 155p.

2. TOURE S.M et Mortelmans J (1991). Impact de la Trypanosomose Animale Africaine. Bull. Scien. Acad. R. Sci. Outre - Mer. 36 (1990-2) : 257p

3. Djitèye et al .1997. Djitèye et al. 1997 : Réactualisation des données sur la répartition des glossines au Mali. Rév. Elev. Méd. Vét. Pays Trop. , 50 (2) : $126-132$.

4. Cuisance et Politzar, 2003. Répartition des glossines dans la zone pastorale de Sideradougou, Burkina. Revue Elev. Med. Vet. Pays trop. 37 : 99 $-113$
5. AGETIC, 2011. Données géographiques gouvernorat de la région de Sikasso.

6. Challier A - laveissière $C, 1973$. Un nouveau piège pour la capture des glossines. Revue Elev. Vet. Pays tropicaux, $37: 31-59$

7. CIRDES, 2002. Fiche technique : la dissection des glossines ou mouches tsé-tsé.

8. BASS et al. 2010. Répartition des glossines et autres mouches piqueuses dans le bassin du Bani au Mali. Editions Universitaires Européennes, Juillet 2011. $3-60$.

$\mathrm{N}^{\circ}$ ISBN: $978-613-1-56145-0$. Pages

9. BASS et al. 2014. Impact du changement global sur la répartition spatiale des glossines dans le cercle de Bougouni au Mali.

Revue Malienne d'Infectiologie et de Microbiologie, 2014, Tome 1, Pages 28 - 33.

10. Jamonneau et al. 2004 : Analyse des groupes d'âge physiologique des glossines. Cah. IRD, ser. Ent. Méd. Et Parasitol., vol. xx, n 3 : 189 $-197$

11. Dao et al. 2008. Impact de la sécheresse et de la dégradation des aires protégées sur la répartition des trypanosomoses bovines et de leurs vecteurs dans le bassin versant de l'Oti au nord du Togo. Revue Elev. Méd. Vét. Pays trop. , 2008, 61 (3-4) : 153-160

12. De La Rocque et al. 2005 : A model of diffusion of Glossina palpalis gambiensis in Burkina Fao. Revue Elev. Méd. Vét. Pays trop. , 2005 
Tableau I : Les villages visités dans le cercle de kadiolo et leurs coordonnées géographiques.

\begin{tabular}{|l|c|}
\hline Villages & Coordonnées géographiques \\
\hline Fanidiama & $1049312 ; 0556419$ \\
\hline Zégoua & $1052270 ; 0558078$ \\
\hline Katele & $1063138 ; 0561703$ \\
\hline Nassoulou & $1054232 ; 0559783$ \\
\hline Zekoun & $1039562 ; 0599187$ \\
\hline N'Goko & $1041361 ; 0603455$ \\
\hline Nafegue & $1058820 ; 0598953$ \\
\hline Diou & $1058770 ; 0596945$ \\
\hline Dioumatene & $1053230 ; 0590649$ \\
\hline Galamakourou & $1067339 ; 0615000$ \\
\hline Kamberegué & $1062692 ; 0606383$ \\
\hline Gninasso & $1054052 ; 0570469$ \\
\hline Lofigué & $1063985 ; 05698880$ \\
\hline Daoulasso & $1091947 ; 0570365$ \\
\hline Kaï & $1096972 ; 0568750$ \\
\hline Niérouani & $1083274 ; 0563250$ \\
\hline Siekourani & $1073821 ; 0560785$ \\
\hline Nimbougou & $1085612 ; 0550526$ \\
\hline Bananso & $1087927 ; 0603161$ \\
\hline Siranikoroba & $1078178 ; 0549377$ \\
\hline & \\
\cline { 2 - 2 } & \\
\cline { 2 - 2 } & \\
\hline
\end{tabular}

Tableau II : Les villages visités dans le cercle de Sikasso et leurs coordonnées géographiques.

\begin{tabular}{|l|c|}
\hline Villages & Coordonnées géographiques \\
\hline Konzasso - Diassa & $1096967 ; 0583631$ \\
\hline Nagnasso & $1117371 ; 0586982$ \\
\hline Kitagalasso & $1113587 ; 0586806$ \\
\hline Pissanso & $1110190 ; 0594337$ \\
\hline M'Belasso & $1113604 ; 0595335$ \\
\hline Goloniena & $1109374 ; 0590827$ \\
\hline Tiogola & $1100827 ; 0594688$ \\
\hline Kapala & $1110204 ; 0579341$ \\
\hline Diassadiè & $1111692 ; 0571955$ \\
\hline Ouahibera & $1102923 ; 0559338$ \\
\hline Finibougou & $1106717 ; 0566069$ \\
\hline Mandela & $1119382 ; 0550851$ \\
\hline Farako & $1115881 ; 0552726$ \\
\hline Kafela & $1023779 ; 0548258$ \\
\hline \hline
\end{tabular}




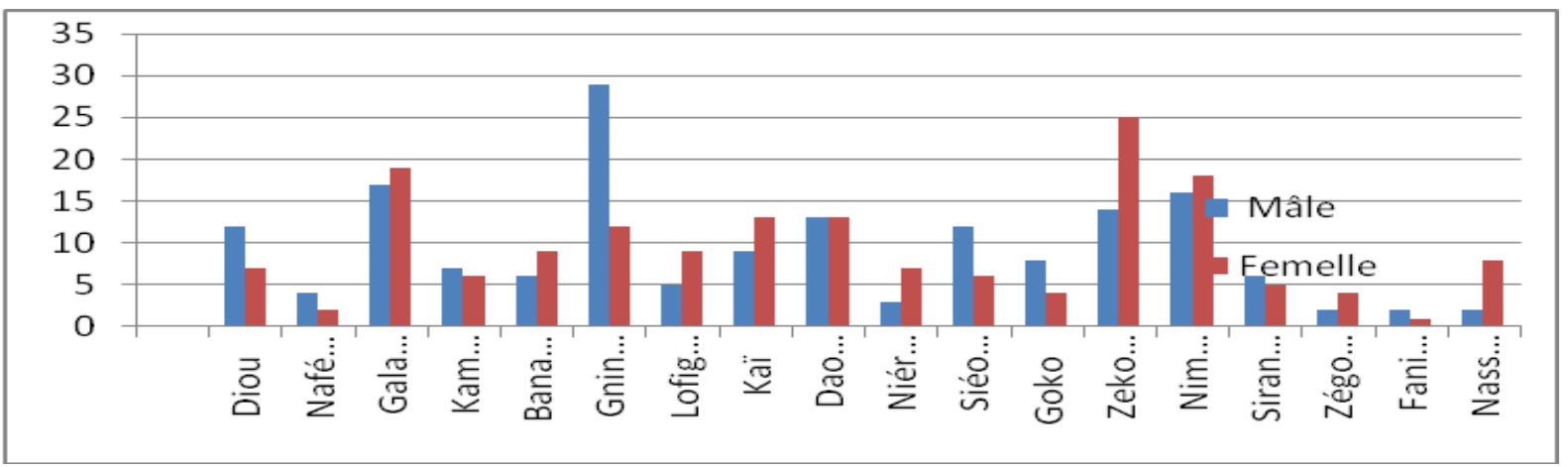

Figure 1 : Nombre de Glossina palpalis gambiensis capturé dans les villages concernés par l'étude dans le cercle de Kadiolo.

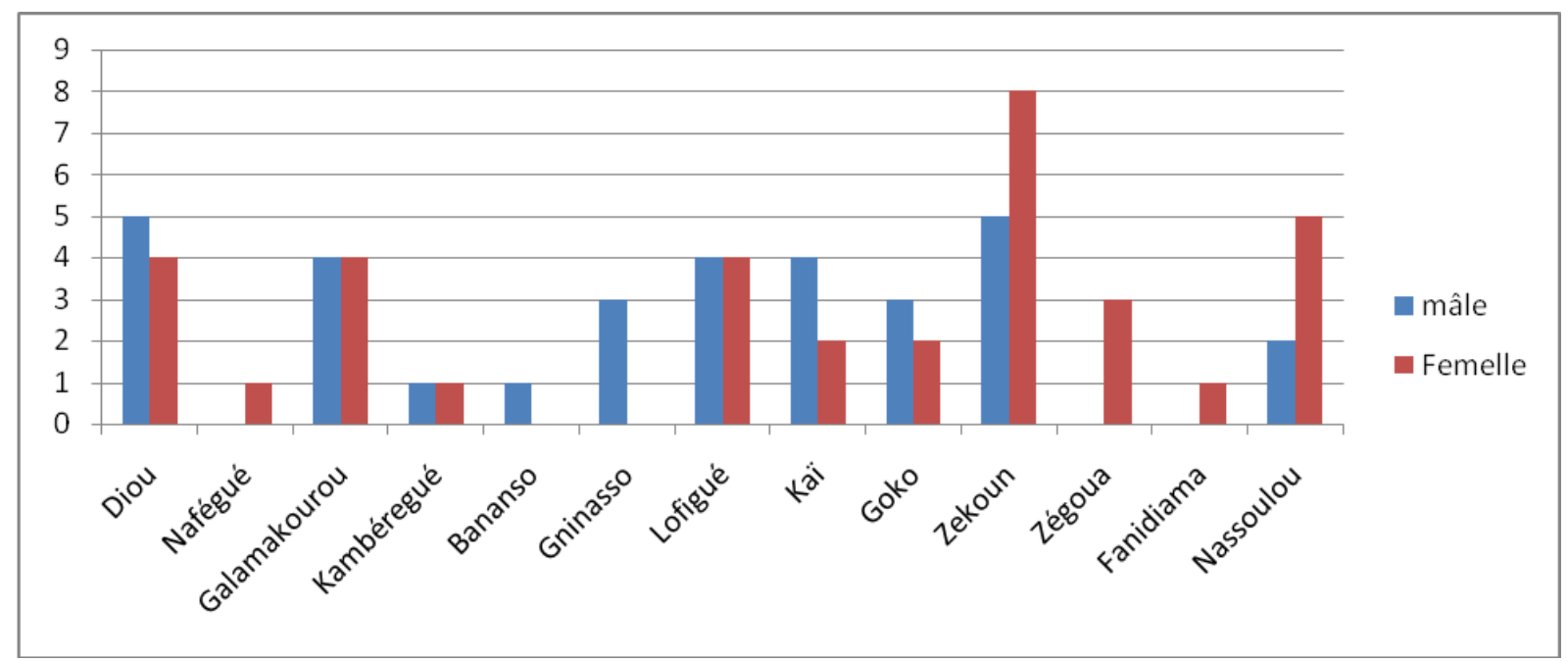

Figure 2 : Nombre de Glossina tachinoides capturé dans les différents villages de l'étude dans le cercle de Kadiolo.

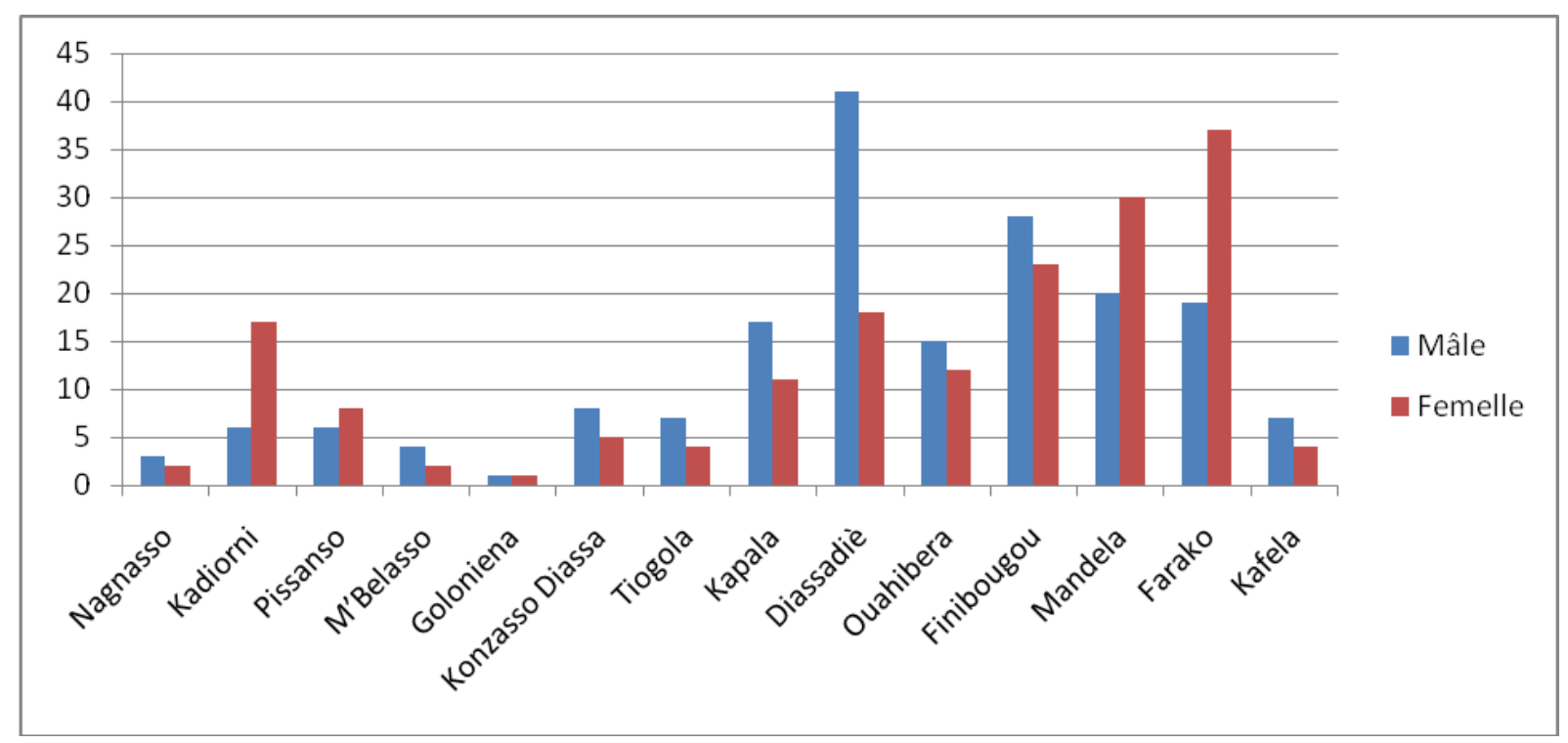

Figure 3 : Nombre de Glossina palpalis gambiensis capturé dans le cercle de Sikasso. 


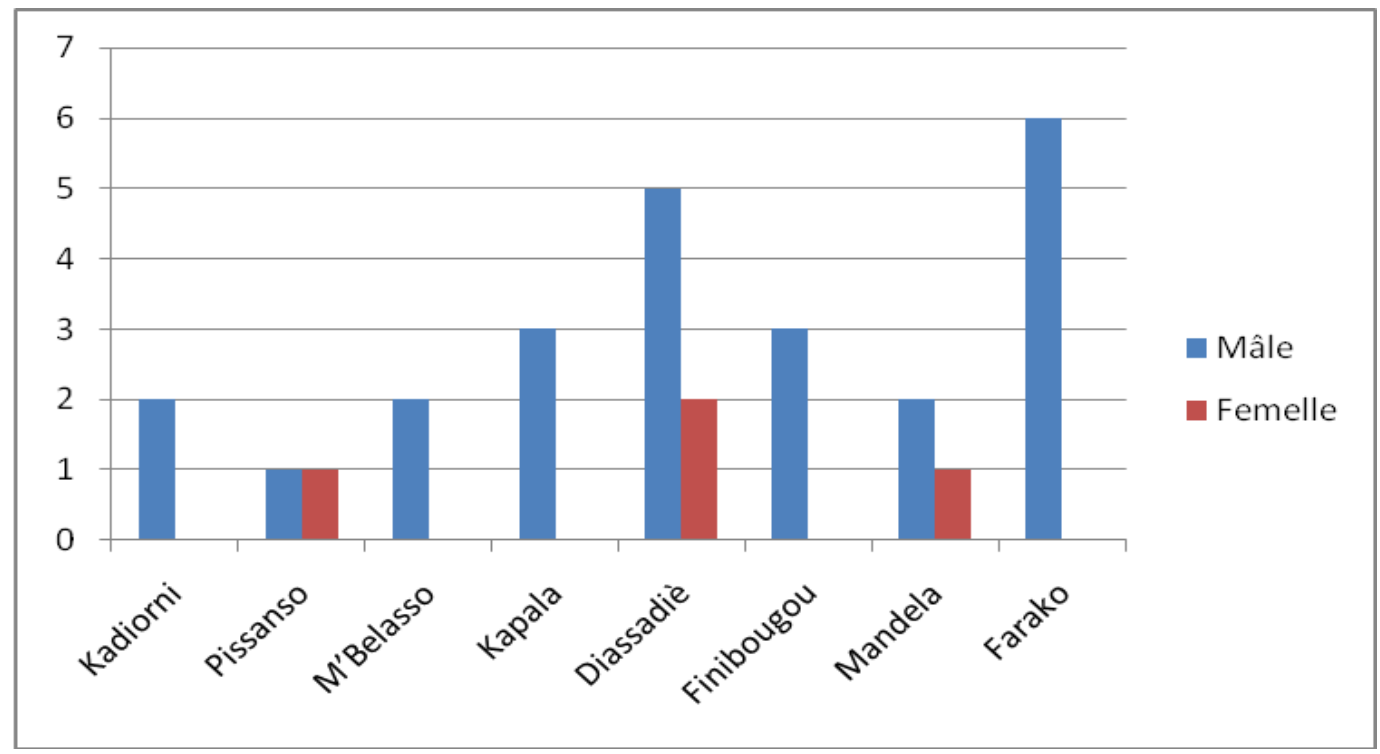

Figure 4 : Nombre de Glossina tachinoïdes capturé dans le cercle de Sikasso.

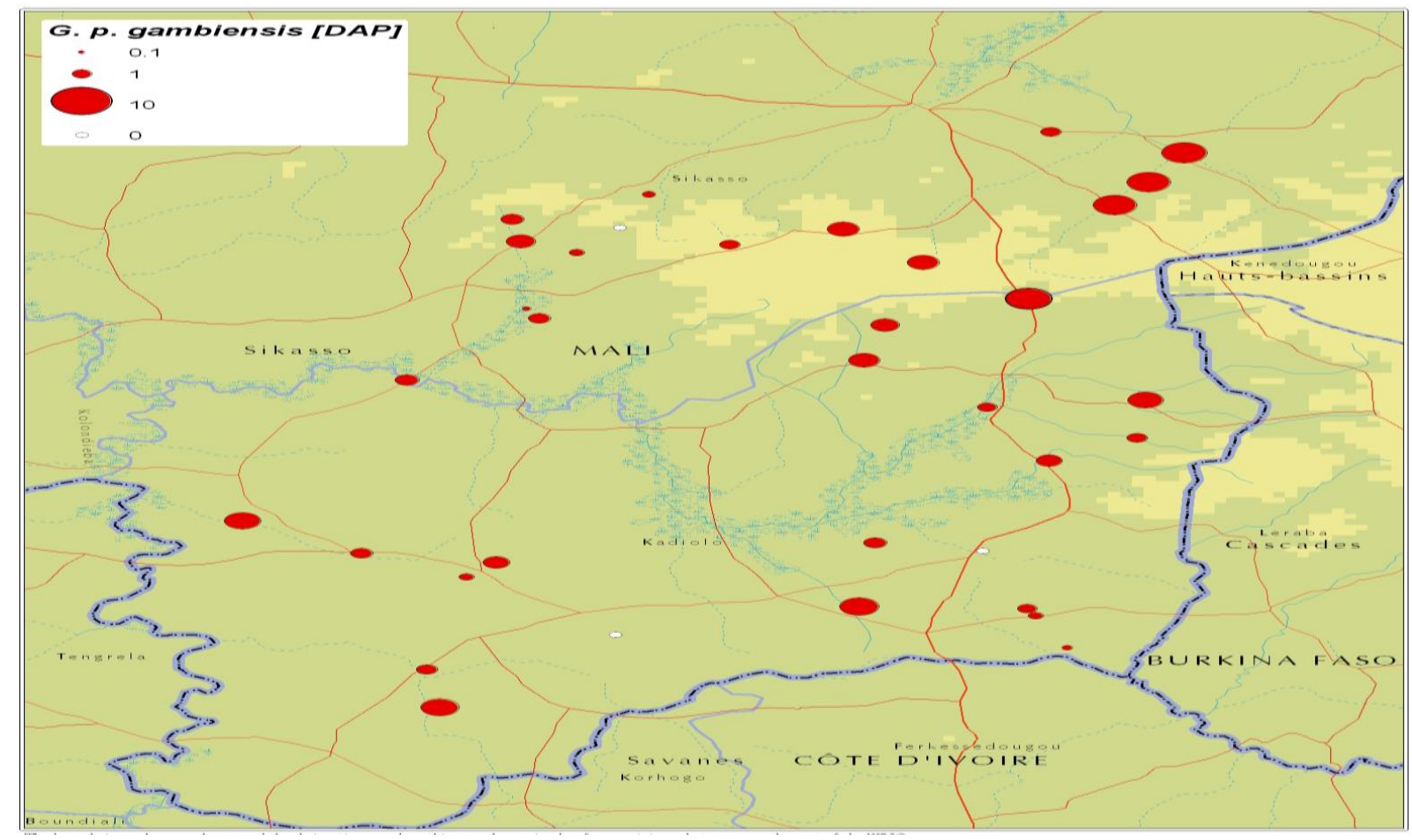

Figure 5 : DAP de Glossina palpalis gambiensis dans les cercles de Kadiolo et Sikasso (Source :

Giuliano CECCHI) 


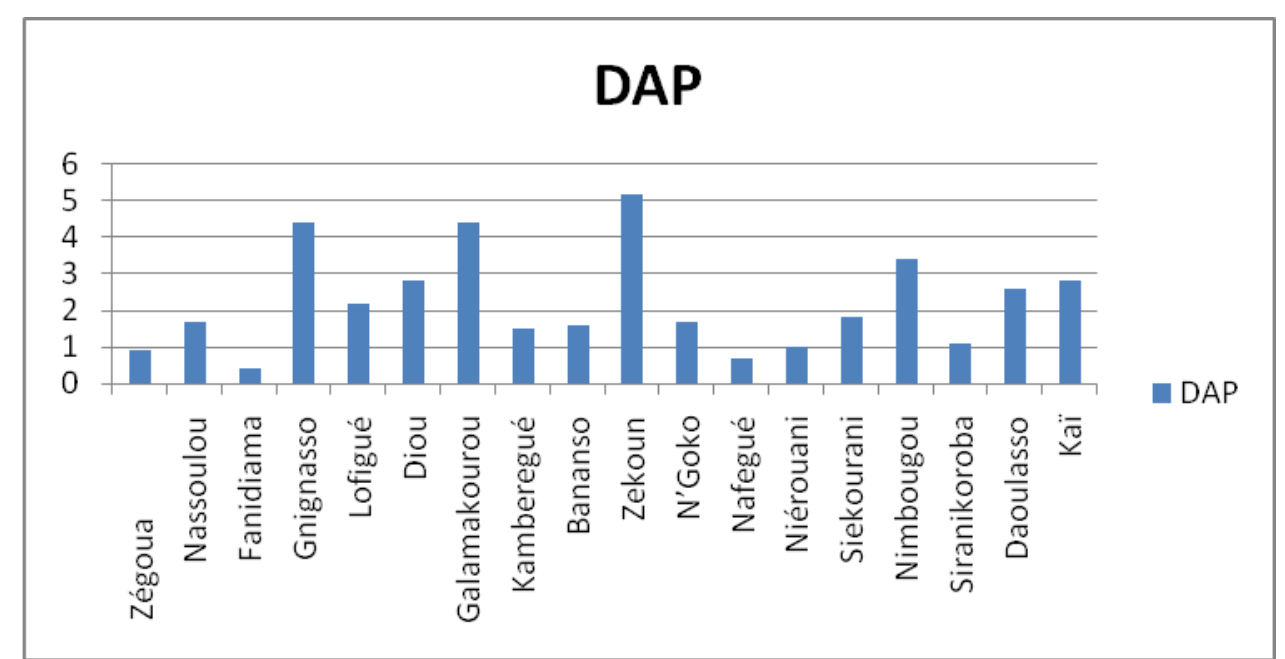

Figure 6 : DAP des glossines dans le cercle de Kadiolo.

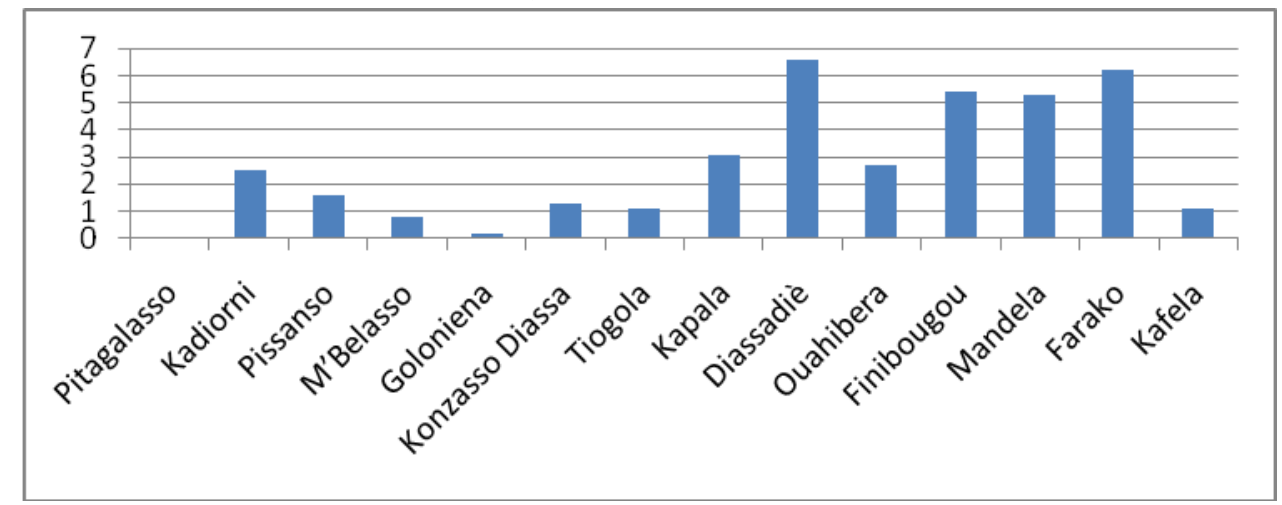

Figure 7 : DAP des glossines dans le cercle de Sikasso.

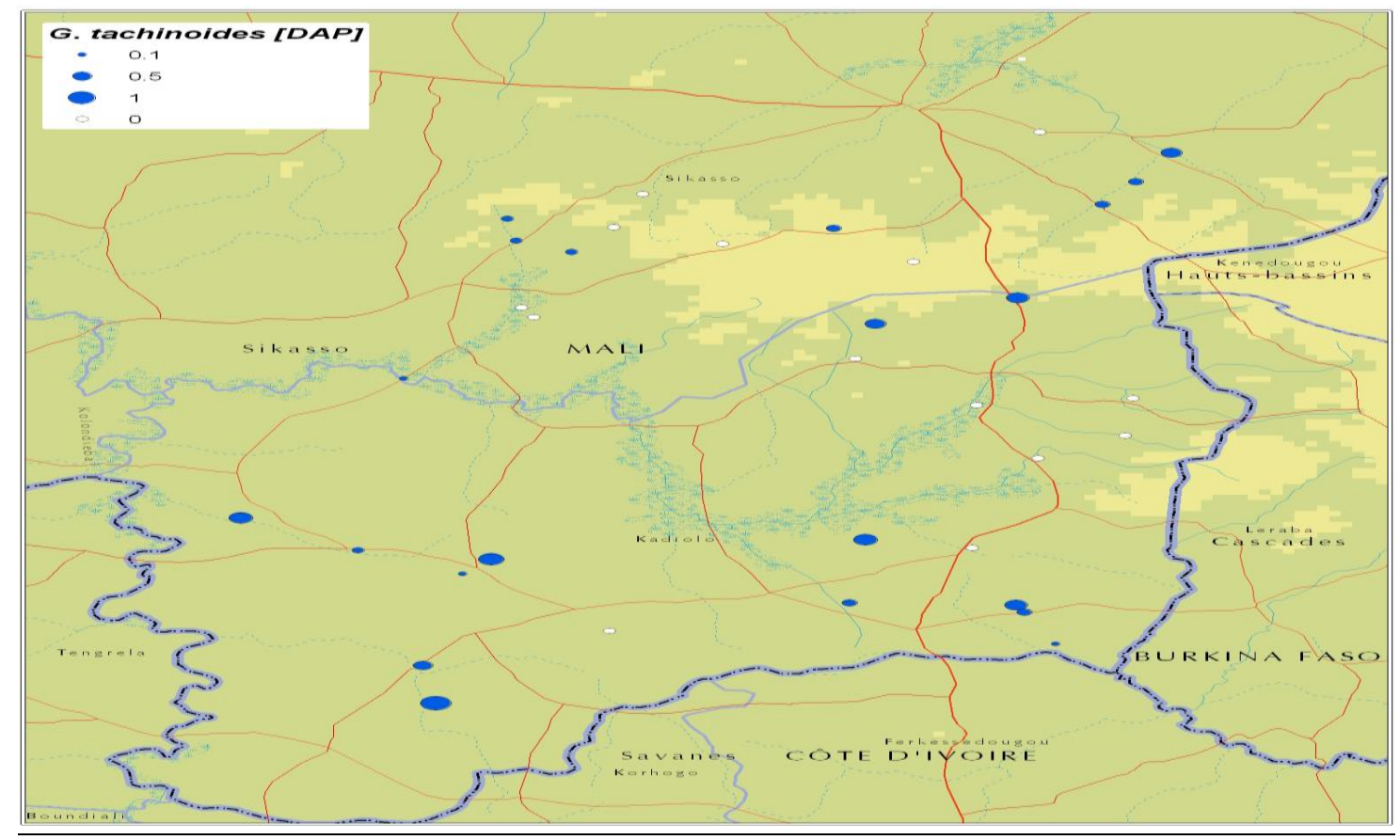

Figure 8: DAP de Glossina tachinoïdes dans les cercles de Kadiolo et Sikasso. (Source : Giuliano CECCHI) 
Tableau III : Sexe ratio des glossines capturées.

\begin{tabular}{|c|c|}
\hline Villages & Sex-ratio \\
\hline Zégoua & 0,28 \\
\hline Nassoulou & 0,30 \\
\hline Fanidiama & 1 \\
\hline Katele & 0 \\
\hline Gninasso & 2,66 \\
\hline Lofigué & 0,69 \\
\hline Diou & 1,54 \\
\hline Dioumatene & 0 \\
\hline Galamakourou & 0,91 \\
\hline Kamberegue & 1,14 \\
\hline Bananso & 0,77 \\
\hline Zekoun & 0,57 \\
\hline N'Goko & 1,83 \\
\hline Nafegué & 1,33 \\
\hline Niérouani & 0,42 \\
\hline Sieou-Kourani & 2 \\
\hline Nimbougou & 0,88 \\
\hline Siranikoroba & 1,2 \\
\hline Daoulasso & 1 \\
\hline Каї & 0,86 \\
\hline Nagnasso & 1,5 \\
\hline Pitagalasso & 0 \\
\hline Kadiorni & 0,47 \\
\hline Pissanso & 0,77 \\
\hline M'Belasso & 3 \\
\hline Goloniena & 1 \\
\hline Konzasso Diassa & 1,6 \\
\hline Tiogola & 1,75 \\
\hline Kapala & 1,81 \\
\hline Diassadiè & 2,3 \\
\hline Ouahibera & 1,25 \\
\hline Finibougou & 1,34 \\
\hline Mandela & 0,70 \\
\hline Farako & 0,67 \\
\hline Kafela & 1,75 \\
\hline Total & 1,12 \\
\hline
\end{tabular}


Tableau IV : Résultats de capture des autres mouches piqueuses du cercle de Kadiolo.

\begin{tabular}{|l|c|c|}
\hline Localités & Autres mouches piqueuses & Nombre \\
\hline Zégoua & Tabanus gratus & 0 \\
\hline Fanidiama & Pas de capture & 0 \\
\hline Katele & Pas de capture & 0 \\
\hline Nassoulou & Pas de capture & 1 \\
\hline Gninasso & Atylotus agrestis & 0 \\
\hline Lofigué & Pas de capture & 1 \\
\hline Diou & Atylotus agrestis & 0 \\
\hline Dioumantene & Pas de capture & 0 \\
\hline Galamakourou & Pas de capture & 1 \\
\hline Kamberegue & taenicola & 2 \\
\hline Zekoun & Atylotus agrestis & 1 \\
\hline N'Goko & Tabanus gratus & 0 \\
\hline Nafegué & Pas de capture & 0 \\
\hline Niérouani & Pas de capture & 0 \\
\hline Siekourani & Pas de capture & 0 \\
\hline Nimbougou & Pas de capture & 0 \\
\hline Siranikoroba & Pas de capture & 0 \\
\hline Daoulasso & Pas de capture & 0 \\
\hline Kaï & Pas de capture & 0 \\
\hline Bananso & Pas de capture & 17 \\
\hline TOTAL & Tabanus gratus & 24 \\
\hline
\end{tabular}


Tableau V : Résultats de capture des autres mouches piqueuses du cercle de Sikasso.

\begin{tabular}{|l|c|c|}
\hline Localités & Autres mouches piqueuses & Nombre \\
\hline Nagnasso & Pas de capture & 0 \\
\hline Pitagalasso & Pas de capture & 0 \\
\hline Kadiorni & Pas de capture & 1 \\
\hline Pissanso & Tabanus gratus & 1 \\
\hline M'Belasso & Tabanus taneola & 0 \\
\hline Goloniena & Pas de capture & 4 \\
& Tabanus gratus & 1 \\
\hline Konzasso Diassa & Atylotus agrestis & 4 \\
\hline Tiogola & Tabanus gratus & 3 \\
\hline Kapala & Tabanus gratus & 0 \\
\hline Diassadiè & Pas de capture & 0 \\
\hline Ouahibera & Pas de capture & 0 \\
\hline Finibougou & Pas de capture & 0 \\
\hline Mandela & Pas de capture & 0 \\
\hline Farako & Pas de capture & 0 \\
\hline Kafela & Pas de capture & 0 \\
\hline TOTAL & Pas de capture & 14 \\
\hline
\end{tabular}

